Pure and Applied Mathematics Quarterly

Volume 6, Number 2

(Special Issue: In honor of

Michael Atiyah and Isadore Singer)

$545-554,2010$

\title{
Whitehead's Integral Formula, Isolated Critical Points, and the Enhancement of the Milnor Number
}

Lee Rudolph

\author{
for Is Singer on his $85^{\text {th }}$ birthday
}

\begin{abstract}
J. H. C. Whitehead gave an elegant integral formula for the Hopf invariant $\mathcal{H}(\mathbf{p})$ of a smooth map $\mathbf{p})$ from the 3 -sphere to the 2 -sphere. Given an open book structure $\mathbf{b}$ on the 3 -sphere (or, essentially equivalently, an isolated critical point of a map $F$ from $\mathbb{R}^{4}$ to $\mathbb{R}^{2}$ ), Whitehead's formula can be "integrated along the fibers" to express $\mathcal{H}(\mathbf{p})$ as the integral of a certain 1 -form over $S^{1}$. In case $\mathbf{p}$ is geometrically related to $\mathbf{b}$ (or $F$ )-for instance, if $\mathbf{p}$ is the map (one component of the fiberwise generalized Gauss map of $F$ ) whose Hopf invariant $\lambda(K)$ is the "enhancement of the Milnor number" of the fibered link $K \subset S^{3}$ associated to $F$ (or $\mathbf{b}$ ), previously studied by the author and others - it might be hoped that this 1-form has geometric significance. This note makes that hope somewhat more concrete, in the form of several speculations and questions.
\end{abstract}

Keywords: Enhancement, fibered link, Hopf invariant, Milnor number, open book, Whitehead's integral formula

Received March 7, 2008.

During various parts of the period when this research was performed, the author was partially supported by the Fonds National Suisse, and by NSF awards DMS-8801915, DMS-9504832, DMS-0308894, and IIS-0713335. 


\section{WhiteheAD's INTEGRAL FORMUla AND ISOlATED CRITICAL POINTS}

Let p: $S^{3} \rightarrow S^{2}$ be smooth. According to Whitehead [21] (see also [22, 2]), the Hopf invariant $\mathcal{H}(\mathbf{p})$ of $\mathbf{p}$ can be calculated as follows. Let $\Omega$ be the volume 2-form on $S^{2}$, normalized to give $S^{2}$ volume 1 . Since $\Omega$ is closed, so is its pullback $\Omega_{\mathbf{p}}=\mathbf{p}^{*}(\Omega)$; since $H^{2}\left(S^{3} ; \mathbb{R}\right)=\{0\}, \Omega_{\mathbf{p}}$ is in fact exact. Let $\eta_{\mathbf{p}}$ be a 1 -form on $S^{3}$ with $d \eta_{\mathbf{p}}=\Omega_{\mathbf{p}}$. If $g$ is any 0 -form on $S^{3}$, then also $d\left(\eta_{\mathbf{p}}+d g\right)=\Omega_{\mathbf{p}}$, and since $H^{1}\left(S^{3} ; \mathbb{R}\right)=\{0\}$, every solution $\eta$ of $d \eta=\Omega_{\mathbf{p}}$ is of the form $\eta_{\mathbf{p}}+d g$. Thus, pointwise, the 3 -form $\eta_{\mathbf{p}} \wedge \Omega_{\mathbf{p}}$ depends strongly on the choice of $\eta_{\mathbf{p}}$. Nonetheless, Whitehead showed that its integral is independent of this choice:

$$
\int_{S^{3}} \eta_{\mathbf{p}} \wedge \Omega_{\mathbf{p}}=\mathcal{H}(\mathbf{p}) .
$$

It is clear that WIF remains valid, mutatis mutandis, if subjected to any of various modifications: the target of $\mathbf{p}$ could be $\mathbb{R}^{3} \backslash\{\mathbf{0}\}$ or $\left(\mathbb{R}^{3} \backslash\{\mathbf{0}\}\right) \times \mathbb{R}$, the domain of $\mathbf{p}$ could be a punctured neighborhood of $\mathbf{0}$ in $\mathbb{R}^{4}$, and (in the latter case) the integration in (WIF) could be over any 3 -cycle in the domain of $\mathbf{p}$ dual to the puncture at $\mathbf{0}$.

Let $U$ be a neighborhood of $\mathbf{0}$ in $\mathbb{R}^{4}, F=(f, g): U \rightarrow \mathbb{R}^{2}$ a map with $F(\mathbf{0})=\mathbf{0}$ such that $F$ is smooth on $U \backslash\{\mathbf{0}\}$ and $F$ is continuous at $\mathbf{0}$. We will say that $\mathbf{0}$ is an isolated critical point of $F$ provided that there exists $\delta>0$ such that, if $0<\|\mathbf{x}\| \leq \delta$, then the derivative $D F(\mathbf{x})$ has rank 2; and (following Kauffman \& Neumann [8]) we will say that $\mathbf{0}$ is a tame isolated critical point of $F$ if, further, for all sufficently small $\delta>0$, (a) $F^{-1}(\mathbf{0})$ and $\delta S^{3}$ intersect transversely, and (b) there exists $\varepsilon(\delta)>0$ such that, if $0<\varepsilon \leq \varepsilon(\delta)$, then $F^{-1}\left(\varepsilon D^{2}\right) \cap \delta D^{4}$ is a topological 4-disk that is smooth except for corners along $F^{-1}\left(\varepsilon S^{1}\right) \cap \delta S^{3}$.

Examples. (a) If $F$ is smooth at $\mathbf{0}$ and $\mathbf{0}$ is a regular point of $F$, then $\mathbf{0}$ is a tame isolated critical point of $F$. (b) If $F$ is (the real-polynomial mapping underlying) a complex-polynomial mapping $\left(\mathbb{C}^{2}, \mathbf{0}\right) \rightarrow(\mathbb{C}, 0)$ without repeated factors, then $\mathbf{0}$ is a tame isolated critical point of $F$. The differential topology of this case (and its higher-dimensional generalization), first studied by Milnor [14], continues to be a lively topic of investigation. (c) Let $F:\left(\mathbb{R}^{4}, \mathbf{0}\right) \rightarrow\left(\mathbb{R}^{2}, \mathbf{0}\right)$ be a real-polynomial mapping. For a generic such $F$, the real-algebraic set of critical points of $F$ is 1-dimensional at each of its points, so $\mathbf{0}$ is an isolated critical point of $F$ if and only if it is a regular point. Nonetheless, there exist many 
real-polynomial mappings $F$ for which $\mathbf{0}$ is a non-regular isolated critical point (necessarily tame, [8]). Among these mappings, those that underlie complexpolynomial mappings are neither the only, nor necessarily the most interesting, examples. (A wide variety of essentially non-complex examples are known; see [13, 16, 19, 18, 17, 1, 6].)

Proposition 1. If $F=(f, g): U \rightarrow \mathbb{R}^{2}$ has a tame isolated critical point at $\mathbf{0}$, and $\mathbf{p}: U \backslash\{\mathbf{0}\} \rightarrow \mathbb{R}^{3} \backslash\{\mathbf{0}\}$ is a smooth map, then for all sufficiently small $\delta>0$,

$$
\mathcal{H}(\mathbf{p})=\lim _{\varepsilon \rightarrow 0} \int_{\varepsilon S^{1}} \quad \int \eta_{\mathbf{p}} \wedge \Omega_{\mathbf{p}}
$$

Proof. By hypothesis (b) in the definition of tameness, the 3-manifold-withcorners $\partial\left(F^{-1}\left(\varepsilon D^{2}\right) \cap \delta D^{4}\right)$ (with its natural orientation) is a 3-cycle homologous to $\delta S^{3}$ in $U \backslash\{\mathbf{0}\}$, provided that $\delta$ and $\varepsilon$ are sufficiently small. As a 3-cycle, $\partial\left(F^{-1}\left(\varepsilon D^{2}\right) \cap \delta D^{4}\right)$ is the sum of two 3-chains, $C_{1}(\delta, \varepsilon)=F^{-1}\left(\varepsilon S^{1}\right) \cap \delta D^{4}$ and $C_{2}(\delta, \varepsilon)=F^{-1}\left(\varepsilon D^{2}\right) \cap \delta S^{3}$; the latter, by hypothesis (a) in the definition of tameness, is a neighborhood of a smooth link $L=\delta S^{3} \cap F^{-1}(\mathbf{0})$ in $\delta S^{3}$, and it is clear that for a fixed $\delta$ this neighborhood shrinks down to $L$ as $\varepsilon$ goes to 0 . By the comments after (WIF),

$$
\mathcal{H}(\mathbf{p})=\int_{C_{1}(\delta, \varepsilon)} \eta_{\mathbf{p}} \wedge \Omega_{\mathbf{p}}+\int_{C_{2}(\delta, \varepsilon)} \eta_{\mathbf{p}} \wedge \Omega_{\mathbf{p}},
$$

so

$$
\mathcal{H}(\mathbf{p})=\lim _{\varepsilon \rightarrow 0} \int_{C_{1}(\delta, \varepsilon)} \eta_{\mathbf{p}} \wedge \Omega_{\mathbf{p}}
$$

this becomes (1) after integration along the fiber.

\section{WhiteHEAD'S INTEGRAL FORMULA AND OPEN BOOKS}

Tame isolated critical points are closely related to open-book structures. Here, an open book on $S^{3}$ is a smooth map b: $S^{3} \rightarrow \mathbb{R}^{2}$ such that $\mathbf{0}$ is a regular value of $\mathbf{b}$ and $\mathbf{b} /\|\mathbf{b}\|: S^{3} \backslash \mathbf{b}^{-1}(\mathbf{0}) \rightarrow S^{1}$ is a fibration. Given an open book $\mathbf{b}$, it is easy to check that $\mathbf{0}$ is a tame isolated critical point of $c o(\mathbf{b}): \mathbb{R}^{4} \rightarrow \mathbb{R}^{2}$, where $c o(\mathbf{b})(t \mathbf{x})=t \mathbf{b}(\mathbf{x})$ for all $\mathbf{x} \in S^{3}$ and $t \geq 0$. Conversely [8], if $\mathbf{0}$ is a tame isolated critical point of $F:(U, \mathbf{0}) \rightarrow\left(\mathbb{R}^{2}, \mathbf{0}\right)$, then there is an open book $\mathbf{b}_{F}$ (unique up 
to an obvious equivalence relation) such that the isolated critical points at $\mathbf{0}$ of $F$ and of $c o\left(\mathbf{b}_{F}\right)$ are equivalent (again, in an obvious sense).

The binding of the open book $\mathbf{b}$ is $L_{\mathbf{b}}=\mathbf{b}^{-1}(\mathbf{0})$. The $\theta^{\text {th }}$ page of $\mathbf{b}$ is $S_{\mathbf{b}}(\theta)=$ $\mathbf{b}^{-1}(\{t(\cos (\theta), \sin (\theta)) \mid t \geq 0\})$. It is immediate from the definitions that $L_{\mathbf{b}}$ is a smooth link (naturally oriented by the orientations of $S^{3}$ and $S^{2}$ ) and that each $S_{\mathbf{b}}(\theta)$ is a Seifert surface bounded by $L_{\mathbf{b}}$. In fact, the binding of an open book is a fibered link, and each page is a fiber surface for that link.

Proposition 2. If $\mathbf{b}: S^{3} \rightarrow \mathbb{R}^{2}$ is an open book, and $\mathbf{p}: S^{3} \rightarrow S^{2}$ is a smooth map, then

$$
\mathcal{H}(\mathbf{p})=\lim _{\varepsilon \rightarrow 0} \int_{S^{1}} \underset{S_{\mathbf{b}}(\theta) \backslash \mathbf{b}^{-1}\left(\varepsilon\left(\operatorname{Int} D^{2}\right)\right)}{ } \eta_{\mathbf{p}} \wedge \Omega_{\mathbf{p}}
$$

Proof. This is entirely analogous to Proposition 1.

\section{The enhancement of the Milnor number}

As in $\$ 1$, let $F=(f, g):(U, \mathbf{0}) \rightarrow\left(\mathbb{R}^{2}, \mathbf{0}\right)$ be smooth on $U \backslash\{0\} \subset \mathbb{R}^{4}$ and continuous at $\mathbf{0}$. Easily, $\mathbf{0}$ is an isolated critical point of $F$ if and only if there exists $\delta>0$ such that, if $0<\|\mathbf{x}\| \leq \delta$, then $(d f \wedge d g)(\mathbf{x}) \neq 0$. In this case, the selfdual (resp., anti-self-dual) 2-form $d f \wedge d g+\star(d f \wedge d g$ ) (resp., $d f \wedge d g-\star(d f \wedge d g)$ ), where $\star$ is the Hodge star operator with respect to the flat metric on $\mathbb{R}^{4}$, is non-degenerate; writing

$$
\begin{aligned}
d f \wedge d g \pm \star(d f \wedge d g)= & A_{ \pm}(d x \wedge d y+ \pm d u \wedge d v) \\
& +B_{ \pm}(d x \wedge d u \mp d y \wedge d v)+C_{ \pm}(d x \wedge d v \pm d y \wedge d u),
\end{aligned}
$$

in terms of linear coordinates $(x, y, u, v)$ on $\mathbb{R}^{4}$, non-degeneracy means that $\left(A_{ \pm}, B_{ \pm}, C_{ \pm}\right): \delta D^{4} \backslash\{\mathbf{0}\} \rightarrow \mathbb{R}^{3}$ avoids $\mathbf{0}$, and so has a well-defined Hopf invariant. Define $\lambda(F)=\mathcal{H}\left(A_{+}, B_{+}, C_{+}\right), \rho(F)=\mathcal{H}\left(A_{-}, B_{-}, C_{-}\right)$.

Lemma. $\lambda(F)$ and $\rho(F)$, so defined, equal $\lambda(F)$ and $\rho(F)$ as defined in [19].

Proof. Let $G$ be the Grassmann manifold of oriented 2-planes in (oriented) $\mathbb{R}^{4}$. In [19], $(\lambda(F), \rho(F))$ is defined to be the homotopy class of the field of oriented 2-planes $\operatorname{ker}(D F)$, identified with a pair of integers via a standard choice of homeomorphism between $G$ and $S^{2} \times S^{2}$. The Plücker coordinates of $\operatorname{ker}(D F)$ 
are the $2 \times 2$ minor determinants of the $2 \times 4$ matrix that represents $D F$ in coordinates $(x, y, u, v)$, and each of the two factors of the map $\operatorname{ker}(D F)$ from a punctured neighborhood of $\mathbf{0}$ to $S^{2} \times S^{2}$ is a certain sum or difference of two Plücker coordinates. For instance, the factor whose Hopf invariant is $\lambda$ has coordinates $f_{x} g_{y}-f_{y} g_{x}+f_{u} g_{v}-f_{v} g_{u}, f_{x} g_{u}-f_{y} g_{v}-f_{u} g_{x}+f_{v} g_{y}$, and $f_{x} g_{v}+$ $f_{y} g_{u}-f_{u} g_{y}-f_{v} g_{x}$, which by inspection are identical, respectively, to $A_{+}, B_{+}$, and $C_{+} ;$and similarly for $\rho$.

In particular, if $F$ is tame, then by [19]

$$
\lambda(F)+\rho(F)=\mu\left(L_{\mathbf{b}_{F}}\right)
$$

is the Milnor number of the fibered link $L_{\mathbf{b}_{F}}$ associated to $F$, that is, the first Betti number of its fiber surface. Thus $\lambda, \rho$, and $\mu$ are, among them, only two independent invariants; following [15], we put $\rho$ aside, calling $\lambda(F)$ the enhancement of the fibered link $L_{\mathbf{b}_{F}}$ (or of its Milnor number), and writing $\lambda(L)$ for $\lambda(F)$.

The enhancement of a fibered link has been shown to have a number of interesting and useful properties, summarized in the following proposition. For proofs, see [20] and references cited therein.

Proposition 3. Let $S$ be a fiber surface in $S^{3}, L$ the fibered link $\partial S$.

(A) The enhancement of $L$ is not determined by its homological monodromy, and in particular it is not determined by the Seifert form of $S$.

(B) Let $\operatorname{Mir}(L)$ denote the mirror image of $L$. Then $\lambda(\operatorname{Mir}(L))+\lambda(L)=$ $\mu(L)$.

(C) Let $S$ be a positive Hopf annulus (so that $L$ is two fibers of a Hopf fibration $S^{3} \rightarrow S^{2}$, oriented to have linking number +1$)$. Then $\lambda(L)=0$. Equivalently, if $F: \mathbb{C}^{2} \rightarrow \mathbb{C}:(z, w) \mapsto z w$, then $\lambda(F)=0$.

(D) As follows immediately from (B) and (C), if $S$ is a negative Hopf annulus, then $\lambda(L)=1$. Equivalently, if $F: \mathbb{C}^{2} \rightarrow \mathbb{C}:(z, w) \mapsto z \bar{w}$, then $\lambda(F)=1$.

(E) More generally than $(\mathrm{C})$, if $F:\left(\mathbb{C}^{2}, \mathbf{0}\right) \rightarrow(\mathbb{C}, 0)$ is a complex polynomial without repeated factors, then $\lambda(F)=0$.

(F) If $S$ is a Murasugi sum of fiber surfaces $S_{1}$ and $S_{2}$, then $\lambda(L)=\lambda\left(\partial\left(S_{1}\right)\right)+$ $\lambda\left(\partial\left(S_{2}\right)\right)$. 
(G) More generally than $(\mathrm{F})$, if $F$ unfolds into $F_{1}$ and $F_{2}$, in the sense of [15], then $\lambda(F)=\lambda\left(F_{1}\right)+\lambda\left(F_{2}\right)$.

(H) As follows immediately from (C), (D), and $(\mathrm{F})$, if $S$ is a Hopf-plumbed surface, then $\lambda(L)$ is the number of negative Hopf plumbands; in particular, in this case $0 \leq \lambda(L) \leq \mu(L)$.

(I) If a contact structure is associated to L by Giroux's construction [3, 4], then $\lambda(L)$ is the Hopf invariant of that contact structure; in particular, $\lambda(L)$ can be any integer.

$(\mathrm{J})$ Generalizing $(\mathrm{H}), \lambda(L)$ is the net number of negative Hopf plumbands in any stable Hopf plumbing of $S$ (which exists, by [3, 4]).

$(\mathrm{K})$ Let $e(\beta)$ denote the exponent sum of a braid $\beta \in B_{n}$. Given any $\beta$, construct a link $L$ by adjoining to the closed braid $\widehat{\beta}$ the braid axis together with,

for each component $K$ of $\widehat{\beta}$, a nearby oppositely-oriented longitude $-K^{\prime}$, where the linking number of $K$ and $K^{\prime}$ is unrestricted. It is a beautiful observation of Hirasawa (see [6]) that then $L$ is fibered and $\lambda(L)$ is equal to $n-e(\beta)+1$, the Bennequin number of $\beta$.

\section{INTEGRAL FORMULAS FOR THE ENHANCEMENT}

Applying the results in $\$ 1$ to the definitions in $\$ 3$, we can obtain integral formulas for the enhancement.

If one combines

$$
\Omega_{\left(A_{+}, B_{+}, C_{+}\right)}=\frac{A_{+} d B_{+} \wedge d C_{+}+B_{+} d C_{+} \wedge d A_{+}+C_{+} d A_{+} \wedge d B_{+}}{\left(A_{+}^{2}+B_{+}^{2}+C_{+}^{2}\right)^{3 / 2}}
$$

with the formulas for $A_{+}, B_{+}$, and $C_{+}$given in the Lemma, the easily confirmed formula

$$
\begin{aligned}
A_{+}^{2}+B_{+}^{2}+C_{+}^{2}=\left(f_{x}^{2}+f_{y}^{2}+f_{u}^{2}+f_{v}^{2}\right)\left(g_{x}^{2}+g_{y}^{2}+g_{u}^{2}+g_{v}^{2}\right) & \\
& -\left(f_{x} g_{x}+f_{y} g_{y}+f_{u} g_{u}+f_{v} g_{v}\right)^{2},
\end{aligned}
$$

and $\left(\mathrm{WIF}\right.$, , then - provided one has a method to produce a 1 -form $\eta_{\left(A_{+}, B_{+}, C_{+}\right)}$ (there is no shortage of such methods, some more explicit than others) - one can derive a fearsome-looking formula for $\lambda(F)$ as the integral of what appears to me (perhaps wrongly) to be a pointwise-meaningless 3-form, which I forbear to display here. 
Pressing on, one might take heart from the thought that the 2-form $\Omega_{\left(A_{+}, B_{+}, C_{+}\right)}$ seems to be intimately related to the fibers of $F$ over which $\eta_{\left(A_{+}, B_{+}, C_{+}\right)} \wedge \Omega_{\left(A_{+}, B_{+}, C_{+}\right)}$ is integrated in formula (1). Although this formula might be manipulable into something palatable, I have not yet been able to establish anything along those lines.

A similarly regrettable situation, of high hopes dashed by intractable computations, prevails in the case of open books and formula (2).

Question. Are there, in fact, reasonable and meaningful integral formulas for $\lambda$ in the general case? Such formulas do exist for $\mu$. For instance, in the complex case, and in general dimensions, Kennedy [9] defines a "twisted tangent bundle" $E_{\delta}$ over the fiber $F^{-1}(\delta)(\delta \neq 0)$, with $m^{\text {th }}$ Chern form $c_{m}\left(E_{\delta}\right)$, and (simplifying a formula of Griffiths [5]) proves that

$$
1-\mu(L)=\lim _{\varepsilon \rightarrow 0} \lim _{\delta \rightarrow 0} \int_{F^{-1}(\delta) \cap D_{\varepsilon}^{2 m+2}} c_{m}\left(E_{\delta}\right) .
$$

See also [10, 11, 12]. In all these cases, the integrands are "pointwise-meangingful" insofar as they can be interpreted as various kinds of "curvatures". Does some such curvature interpretation exist for general isolated critical points?

\section{FURThER QUESTIONS}

Even if no "meaningful" integral formula for $\lambda$ can be found in the general case, perhaps there are interesting special cases where one exists. In particular, in light of items $(\mathrm{H})$ and $(\mathrm{I})$ in Proposition 3 , it seems natural to ask for a (geometric or other) characterization of those fibered links $L$ for which $0 \leq \lambda(L) \leq \mu(L)$.

Questions. (a) Can this class of fibered links be characterized using integral formulas? (b) Is this class of fibered links perhaps exactly those for which a mapping $F$ can be found such that all fibers of $F$ are minimal surfaces in $\mathbb{R}^{4}$ ?

Remark. Question (b) is emphatically not a conjecture. The main evidence for the affirmative answer is that, if $F$ is a complex polynomial (or the mirror image of a complex polynomial), then all its fibers, being complex curves, are minimal surfaces. Some reason to think that the techniques of this paper might at least be relevant is that, according to Hoffman \& Osserman [7], a necessary and sufficient condition for all the fibers of $F:(U, \mathbf{0}) \rightarrow\left(\mathbb{R}^{2}, \mathbf{0}\right)$ to be minimal is 
that $\delta D^{4} \backslash\{\mathbf{0}\} \rightarrow G: \mathbf{x} \mapsto \operatorname{ker}(d f \wedge d g)$ be fiberwise (anti-)conformal, which puts strong restrictions on $\left(A_{+}, B_{+}, C_{+}\right)$and its anti-self-dual analogue $\left(A_{-}, B_{-}, C_{-}\right)$. There is a considerable literature on topological restrictions of foliations of 3 manifolds by minimal surfaces, some of which might perhaps be adapted to the investigation of this question.

In 1988, before Walter Neumann used the calculus of splice diagrams to work out an example (published in [15]) of a fibered link $L$ with $\lambda(L)<0$-and well before I noticed that, in fact, such links are plentiful among a family of examples already introduced in [19]-, it seemed reasonable (particularly in light of item $(\mathrm{H})$ of Proposition 3) to wonder whether $\lambda(L)$ might be the dimension of some vectorspace naturally associated to $L$. At that time, Isadore Singer suggested to me that, even if it were never negative, it might nonetheless be better interpreted as the Euler characteristic of some complex (or perhaps the index of some operator), rather than as a dimension. Both suggestions seem even better to me now than they did then, but in 20 years I have made no progress on them. I do have, based on little more than wishful thinking, the notion that such a complex might be constructed by taking note of the complex structures naturally imposed on the fibers of $F$ by their induced metrics; note the connection to the Hoffman-Osserman generalized Gauss map.

Question. Can some reader do better with Singer's suggestions?

\section{REFERENCES}

1. Arnaud Bodin and Anne Pichon, Meromorphic functions, bifurcation sets and fibred links, Math. Res. Lett. 14 (2007), no. 3, 413-422. MR 2318645

2. Raoul Bott and Loring W. Tu, Differential forms in algebraic topology, Graduate Texts in Mathematics, vol. 82, Springer-Verlag, New York, 1982. MR 658304 (83i:57016)

3. Emmanuel Giroux, Géométrie de contact: de la dimension trois vers les dimensions supérieures, Proceedings of the International Congress of Mathematicians, Vol. II (Beijing, 2002), Higher Ed. Press, 2002, pp. 405-414. MR 2004c:53144

4. Emmanuel Giroux and Noah Goodman, On the stable equivalence of open books in threemanifolds, Geom. Topol. 10 (2006), 97-114 (electronic). MR 2207791 (2006k:57046)

5. Phillip A. Griffiths, Complex differential and integral geometry and curvature integrals associated to singularities of complex analytic varieties, Duke Math. J. 45 (1978), no. 3, 427-512. MR 80k:53101 
6. Mikami Hirasawa and Lee Rudolph, Constructions of Morse maps for knots and links, and upper bounds on the Morse-Novikov number, Journal of Knot Theory and its Ramifications (to appear), http://arxiv.org/abs/math.GT/0311134

7. David A. Hoffman and Robert Osserman, The geometry of the generalized Gauss map, Mem. Amer. Math. Soc. 28 (1980), no. 236, iii+105. MR MR587748 (82b:53012)

8. Louis H. Kauffman and Walter D. Neumann, Products of knots, branched fibrations and sums of singularities, Topology 16 (1977), no. 4, 369-393. MR 58 \#7644

9. Gary Kennedy, Griffiths' integral formula for the Milnor number, Duke Math. J. 48 (1981), no. 1, 159-165. MR 82j:32021

10. Rémi Langevin, Courbure et singularités complexes, Comment. Math. Helv. 54 (1979), no. 1, 6-16. MR 80g:14006

11. _ Metric study of the neighbourhood of a singularity, Singularities, Part 1 (Arcata, Calif., 1981), Amer. Math. Soc., Providence, R.I., 1983, pp. 669-676. MR 85e:32011

12. _ Geometry of the neighbourhood of a singularity, Functional analysis, holomorphy and approximation theory (Rio de Janeiro, 1980), North-Holland, Amsterdam, 1982, pp. 235-254. MR 84i:32011

13. Eduard Looijenga, A note on polynomial isolated singularities, Nederl. Akad. Wetensch. Proc. Ser. A 74=Indag. Math. 33 (1971), 418-421. MR 46 \#2694

14. John Milnor, Singular points of complex hypersurfaces, Princeton University Press, Princeton, N.J., 1968, Annals of Mathematics Studies, No. 61. MR 39 \#969

15. Walter Neumann and Lee Rudolph, Unfoldings in knot theory, Math. Ann. 278 (1987), no. 1-4, 409-439. MR 89j:57017a

16. B. Perron, Le nœud "huit" est algébrique réel, Invent. Math. 65 (1981/82), no. 3, 441-451. MR 643562 (84d:57005)

17. Anne Pichon, Real analytic germs f $\bar{g}$ and open-book decompositions of the 3-sphere, Internat. J. Math. 16 (2005), no. 1, 1-12. MR 2115674 (2005j:32034)

18. Anne Pichon and José Seade, Real singularities and open-book decompositions of the 3sphere, Ann. Fac. Sci. Toulouse Math. (6) 12 (2003), no. 2, 245-265. MR 2123256 (2005m:57031)

19. L. Rudolph, Isolated critical points of mappings from $\mathbb{R}^{4}$ to $\mathbb{R}^{2}$ and a natural splitting of the Milnor number of a classical fibered link. I. Basic theory; examples, Comment. Math. Helv. 62 (1987), no. 4, 630-645, updated version available at http://arxiv.org/abs/math.GT/ 0203032, MR 88k:57009

20. K_ Knot theory of complex plane curves, Handbook of knot theory (William Menasco and Morwen Thistlethwaite, eds.), Elsevier B. V., Amsterdam, 2005, pp. 349-427. MR 2179266 (2007b:57016)

21. J. H. C. Whitehead, An expression of Hopf's invariant as an integral, Proc. Nat. Acad. Sci. U. S. A. 33 (1947), 117-123. MR 8,525h

22. Hassler Whitney, Geometric integration theory, Princeton University Press, Princeton, N. J., 1957. MR 19,309c 
Lee Rudolph

Department of Mathematics

Clark University, Worcester MA 01610 USA

E-mail: lrudolph@black.clarku.edu 